\title{
Optimization of quasi-normal eigenvalues for Krein-Nudelman strings
}

\author{
Illya M. Karabash a,* \\ a, ${ }^{*}$ Institute of Applied Mathematics and Mechanics of NAS of Ukraine, \\ R. Luxemburg str. 74, Donetsk, 83114, Ukraine. \\ E-mail addresses: i.m.karabash@gmail.com, karabashi@mail.ru \\ ${ }^{*}$ Corresponding author
}

\begin{abstract}
The paper is devoted to optimization of resonances for Krein strings with total mass and statical moment constraints. The problem is to design for a given $\alpha \in \mathbb{R}$ a string that has a resonance on the line $\alpha+\mathrm{i} \mathbb{R}$ with a minimal possible modulus of the imaginary part. We find optimal resonances and strings explicitly.
\end{abstract}

MSC-classes: 49R05, 78M50, 35P25, 47N50

Keywords: Krein string, resonance optimization, quasi-eigenvalue

\section{Introduction}

Recently the increasing interest in loss mechanisms of structured optical and mechanical systems has given rise to spectral optimization problems for dissipative models involving wave equations in inhomogeneous media, see e.g. [1] and references therein. The question is how to design a system with very high or very low loss of energy for oscillations with frequencies in a given range. The rate of energy decay is closely connected to imaginary parts of eigenvalues of the corresponding non-selfadjoint operator. In the paper, these eigenvalues are called quasi-(normal) eigenvalues. Naively, the closer quasi-eigenvalues to the real axis $\mathbb{R}$, the less the rate of energy decay.

It seems that the systematic study of eigenvalue's maximization and minimization problems associated with self-adjoint elliptic operators was initiated by M.G. Krein [7]. While there exists an extensive literature on spectral optimization associated with self-adjoint elliptic operators, an analytic background for spectral optimization problems involving non-self-adjoint operators is not well developed. A possible explanation for this fact is that, for self-adjoint problems, eigenvalues move on the real line and do not have root eigenfunctions of higher order. This leads to a relatively simple statement of the optimization problem and to a relatively simple perturbation theory. Quasieigenvalues' behavior is much more complex. 
The goal of the present paper is to study quasi-eigenvalue optimization problems analytically for Krein strings with dissipation at one end.

In the settings of this paper, a (Krein) string is the interval $(-\infty, 1]$ carrying a dispersed mass, which is represented by a locally bounded nonnegative Borel measure $\mathrm{d} M$. We will speak about the string $\mathrm{d} M$ because the string is completely determined by the measure. If the closed support supp $\mathrm{d} M$ of the measure is finite, the string is called regular and, by definition, the left end $a_{1}$ of a string is the left end of $\operatorname{supp} \mathrm{d} M$. In the trivial case when $\mathrm{d} M$ is the zero measure, we put $a_{1}=1$. We denote the class of regular strings by $\mathfrak{S}_{\text {fin }}$.

The quasi-eigenvalue problem for a regular string

$$
-\frac{\mathrm{d}^{2}}{\mathrm{~d} M \mathrm{~d} x} y(x)=\kappa^{2} y(x), \quad \partial_{x}^{-} y\left(a_{1}\right)=0, \quad y(1)=\frac{i}{\kappa} \partial_{x}^{+} y(1)
$$

is considered. Here the Krein-Feller differential expression $\frac{\mathrm{d}^{2}}{\mathrm{~d} M \mathrm{~d} x}$ can be understood in the integral sense, and $\partial_{x}^{+(-)}$is a properly defined right-hand (resp., left-hand) derivative (see e.g. [6, 2, 4] and Section 2 for details). Problem (1.1) corresponds to free transverse harmonic oscillations of a string with the left end sliding without friction and the right end $x=1$ with friction proportional to the velocity of motion.

Eigen-parameters $\kappa \in \mathbb{C}$ such that (1.1) has a nonzero solution $y$ will be called quasi-eigenvalues. Corresponding eigenfunctions $y$ are called quasi-normal modes. The real part $\alpha=\operatorname{Re} \kappa$ of the quasieigenvalue is the frequency of oscillations corresponding to the quasi-normal mode $y$, the imaginary part $\beta=\operatorname{Im} \kappa$ is always positive and characterizes the rate of decay of the oscillations.

Several other names for $\kappa$ are used, sometimes in slightly different settings: dissipation frequencies [9, 11], resonances and quasi-normal levels (in connection with the time-independent Schrödinger equation and in the Physics literature).

The set of quasi-eigenvalues $\kappa$ of the string $\mathrm{d} M$ is denoted by $K(\mathrm{~d} M)$. It is known that $K(\mathrm{~d} M) \subset$ $\mathbb{C}_{+}$, that quasi-eigenvalues are isolated, and that $\infty$ is their only possible accumulation point (see e.g. [9, 11] and Section 3).

Constraining for regular strings the total mass

$$
\operatorname{Tm}_{M}:=\int_{(-\infty, 1]} \mathrm{d} M
$$

and the statical moment with respect to (w.r.t.) the right end

$$
\operatorname{Stm}_{M}:=\int_{-\infty}^{1}(1-x) \mathrm{d} M,
$$

we define for $m, S>0$ the admissible family

$$
A d=A d(m, S):=\left\{\mathrm{d} M \in \mathfrak{S}_{\text {fin }}: 0<\operatorname{Tm}_{M} \leq m, 0 \leq \operatorname{Stm}_{M} \leq S\right\} .
$$

The optimization problem is

(i) for $\alpha \in \mathbb{R}$, to find

$$
\mathcal{I}(\alpha):=\inf \{\operatorname{Im} \kappa: \operatorname{Re} \kappa=\alpha \text { and } \kappa \in K(\mathrm{~d} M) \text { for certain } \mathrm{d} M \in A d\}
$$


(ii) to find all the strings $\mathrm{d} M$ such that $\alpha+\mathrm{i} \mathcal{I}(\alpha) \in K(\mathrm{~d} M)$, or, if such a string does not exist, to find a sequence of strings $\left\{\mathrm{d} M^{(n)}\right\}_{n=1}^{\infty} \subset A d$ such that $\alpha+\mathrm{i} \beta_{n} \in K\left(\mathrm{~d} M^{(n)}\right)$ with $\beta_{n} \rightarrow \mathcal{I}(\alpha)+0$ as $n \rightarrow \infty$.

In the case when there exists $\mathrm{d} M \in A d$ such that $\alpha+\mathrm{i} \mathcal{I}(\alpha) \in K(\mathrm{~d} M)$ (i.e., when the minimum in (1.3) is achieved), we call $\kappa^{[\alpha]}:=\alpha+\mathrm{i} \mathcal{I}(\alpha)$ optimal quasi-eigenvalue and call $\mathrm{d} M$ optimal string for the frequency $\alpha$.

In Section 2, we solve this optimization problem. It occurs that for $\alpha \notin\left(-S^{-1 / 2}, S^{-1 / 2}\right)$ the infimum in (1.3) is not achieved and optimal strings do not exist. For $\alpha \in\left(-S^{-1 / 2}, S^{-1 / 2}\right)$ the optimal strings exist and we find them and corresponding optimal quasi-eigenvalues explicitly. It occurs that optimal strings $\mathrm{d} M$ consist of a single atom mass placed such that one of the equalities $\mathrm{Tm}_{M}=m$ and $\mathrm{Stm}_{M}=S$ hold, see Theorems 2.4. In other words, optimal strings $\mathrm{d} M$ are extreme points of $A d$. Section 3 is preparative for the proofs of these results. The proofs are given in Section 4.

We use the fact that for the class of strings with finite $\mathrm{Tm}_{M}$ and $\mathrm{Stm}_{M}$ a complete solution of the corresponding direct spectral problem was obtained by Krein and Nudelman [11. While there exist a number of papers on the direct and inverse spectral problems for quasi-eigenvalues (see e.g. [3, 5, 13]), it seems that certain strong additional conditions on $\mathrm{d} M$ and the friction coefficient are always involved. In the author's opinion, the study of the quasi-eigenvalue direct spectral problem for absolutely continuous $\mathrm{d} M$ with densities $M^{\prime}(x)$ in $L^{p}$-spaces could help to understand better the related optimization problems.

Notation. $\mathbb{C}_{ \pm}=\{z \in \mathbb{C}: \pm \operatorname{Im} z>0\}, \mathbb{R}_{ \pm}=\{x \in \mathbb{R}: \pm x>0\}, \mathbb{D}_{\epsilon}(\zeta):=\{z \in \mathbb{C}:|z-\zeta|<\epsilon\}$, $\mathbb{T}_{\epsilon}(\zeta)=\{z \in \mathbb{C}:|z-\zeta|=\epsilon\}$. For $\Omega \subset \mathbb{C}, v_{0}, z \in \mathbb{C}$, let $z \Omega+v_{0}:=\left\{z v+v_{0}: v \in \Omega\right\}$.

\section{Optimal strings and quasi-eigenvalues.}

Following the settings of [11] with some minor changes (see also [4, Ch. 5]), we consider a finite or semi-infinite (Krein) string $\mathrm{d} M$ on $(-\infty, 1]$ with a finite statical moment (first moment) w.r.t. the right end $x=1$, i.e., $\operatorname{Stm}_{M}=\int_{(-\infty, 1]}(1-x) \mathrm{d} M<\infty$. This class of strings is denoted by $\mathfrak{S}$. Then the total mass of the string is finite, $\operatorname{Tm}_{M}:=\int_{(-\infty, 1]} \mathrm{d} M<\infty$. Through the standard procedure the nondecreasing function $M(x):=\int_{(-\infty, x]} d M$ can be associated with the Borel measure $\mathrm{d} M$. By definition, the left end of the string is

$$
a_{1}:=\inf \{x: M(x)>0\} \quad(\geq-\infty)
$$

When $M(x)=0$ on $(-\infty, 1]$, we put $a_{1}=1$. Strings with finite $a_{1}$ are called regular, with $a_{1}=-\infty$ singular. The class of regular strings is denoted by $\mathfrak{S}_{\text {fin }}$.

The quasi-eigenvalue problem for strings is given by (1.1). It was noticed in [9, 11] that it is convenient to include the case $a_{1}=-\infty$ (i.e., singular strings) into the study of problem (1.1) defining the left-hand derivative at $-\infty$ by $\partial_{x}^{-} y(-\infty):=\lim _{x \rightarrow-\infty} \partial_{x}^{-} y(x)$. To define the right-hand derivative $\partial_{x}^{+} y(1)$, we assume that the function $M$ is continued to $x \in(1,+\infty)$ by $M(x):=M(1)+(x-1)$ (i.e., $\mathrm{d} M$ is continued by the Lebesgue measure) and that $y$ satisfies $\frac{\mathrm{d}^{2}}{\mathrm{~d} M \mathrm{~d} x} y(x)+\kappa^{2} y(x)=0$ in a vicinity of $x=1$. Taking this into account, put

$$
a_{2}:=\inf \{x \in \mathbb{R}: \mathrm{d} M(s)=\mathrm{d} s \text { on }[x,+\infty)\} .
$$


Clearly, $-\infty<a_{2} \leq 1$ and $a_{1} \leq a_{2}$. If $a_{2}=1$, the string $\mathrm{d} M$ is called reduced.

Considering the problem in the weighted Hilbert space $L^{2}(-\infty, 1 ; \mathrm{d} M)$ with the norm $\|f\|_{L^{2}(\mathrm{~d} M)}=$ $\left(\int_{(-\infty, 1]}|f|^{2} \mathrm{~d} M\right)^{1 / 2}$ (i.e., assuming $y$ and $\frac{\mathrm{d}^{2}}{\mathrm{~d} M \mathrm{~d} x} y$ in $\left.L^{2}(-\infty, 1 ; \mathrm{d} M)\right)$, we call the eigen-parameters $\kappa$ corresponding to nonzero solutions of (1.1) quasi-eigenvalues of $\mathrm{d} M$ and denote the set of quasieigenvalues by $K(\mathrm{~d} M)$.

It is not difficult to prove that $K(\mathrm{~d} M) \subset \mathbb{C}_{+}$for all $\mathrm{d} M \in \mathfrak{S}$. It occurs that $K(\mathrm{~d} M)$ is the set of zeroes of the entire function

$$
F(z)=F(z ; \mathrm{d} M):=\varphi(1, z)-\mathrm{i} \partial_{x}^{+} \varphi(1, z) / z, \quad z \in \mathbb{C}
$$

where $\varphi(x, z)=\varphi(x, z ; \mathrm{d} M)$ is the solution of the initial value problem

$$
\frac{\mathrm{d}^{2}}{\mathrm{~d} M \mathrm{~d} x} \varphi(x, z)=-z^{2} \varphi(x, z), \quad \varphi\left(a_{1}, z\right)=1, \quad \partial_{x}^{-} \varphi\left(a_{1}, z\right)=0 .
$$

In the case $a_{1}=-\infty$, it is assumed that $\varphi(-\infty, z):=\lim _{x \rightarrow-\infty} \varphi(x, z)$ and the existence of $\varphi$ follows from the theory of Krein strings (see [6, 9, 11] for details and references).

It is obvious that all modes $y$ corresponding to $\kappa \in K(\mathrm{~d} M)$ are equal to $\varphi(\cdot, \kappa ; \mathrm{d} M)$ up to a multiplication by a constant. So the geometric multiplicity of any quasi-eigenvalue equals 1 . In the following, the multiplicity of a quasi-eigenvalue means its algebraic multiplicity.

Definition 2.1 ([9, 11]). The multiplicity of a quasi-eigenvalue is its multiplicity as a zero of the entire function $F(\cdot)$. A quasi-eigenvalue is called simple if its multiplicity is 1 .

For regular strings, this is classical M.V. Keldysh's definition of multiplicity for eigenvalue problems with an eigen-parameter in boundary conditions (see e.g. [12, Sec. 1.2.2-3]).

By $K_{r}(\mathrm{~d} M)$ we denote the set of quasi-eigenvalues of multiplicity $r \in \mathbb{N}$. Each quasi-eigenvalue has a finite multiplicity.

Let us introduce the set $K(A d):=\bigcup_{\mathrm{d} M \in A d} K(\mathrm{~d} M)$ of all possible quasi-eigenvalues for strings from Ad. Recall that the admissible family $A d$ defined by (11.2). Then the function $\mathcal{I}$ given by (1.3) can be written in the form

$$
\mathcal{I}(\alpha)=\inf \{\operatorname{Im} \kappa: \operatorname{Re} \kappa=\alpha \text { and } \kappa \in K(A d)\}
$$

Theorem 2.1. $K(A d)=\mathrm{i}\left[m^{-1},+\infty\right) \cup\left(\mathbb{C}_{+} \backslash\left[\mathbb{D}_{m^{-1}}\left(\mathrm{i} m^{-1}\right) \cup \mathbb{D}_{S^{-1 / 2}}(0)\right]\right)$.

We postpone the proof of this result to Section 4 and turn to its immediate corollary.

Corollary 2.2. If $S \leq m^{2} / 4$, then $\mathcal{I}(\alpha)=\left\{\begin{array}{ll}m^{-1}, & \text { for } \alpha=0 \\ \sqrt{S^{-1}-\alpha^{2}}, & \text { for } 0<|\alpha| \leq S^{-1 / 2} \\ 0, & \text { for } \alpha \geq S^{-1 / 2}\end{array}\right.$.

$$
\text { If } S>m^{2} / 4, \text { then } \mathcal{I}(\alpha)=\left\{\begin{array}{ll}
m^{-1}, & \text { for } \alpha=0 \\
m^{-1}+\sqrt{m^{-2}-\alpha^{2}}, & \text { for } 0<|\alpha|<\sqrt{S^{-1}-m^{2} S^{-2} / 4} \\
\sqrt{S^{-1}-\alpha^{2}}, & \text { for } \sqrt{S^{-1}-m^{2} S^{-2} / 4} \leq|\alpha| \leq S^{-1 / 2} \\
0, & \text { for } \alpha \geq S^{-1 / 2}
\end{array} .\right.
$$


By $\mathrm{d} \Delta_{x_{0}, A}$ we denote the string consisting of an atom mass $A>0$ placed at a point $x_{0} \leq 1$, i.e., writing with Dirac's $\delta$-function, $\mathrm{d} \Delta_{x_{0}, A}=A \delta\left(x-x_{0}\right) \mathrm{d} x$.

The following proposition can be obtained by direct calculations.

Proposition 2.3. Let $x_{0} \leq 1$ and $A>0$. Then the sets of quasi-eigenvalues of the strings $\mathrm{d} \Delta_{x_{0}, A}$ have the following description (taking multiplicities into account):

(i) For $x_{0}<1, K\left(\mathrm{~d} \Delta_{x_{0}, A}\right)=\left\{\mathrm{i} \frac{1}{2\left(1-x_{0}\right)} \pm \sqrt{\frac{1}{A\left(1-x_{0}\right)}-\frac{1}{4\left(1-x_{0}\right)^{2}}}\right\}$.

(ii) In the case $x_{0}=1, K\left(\mathrm{~d} \Delta_{1, A}\right)=\left\{\mathrm{i} A^{-1}\right\}$.

Remark 2.1. In the case $4\left(1-x_{0}\right)=A$, the proposition means that $\frac{\mathrm{i}}{2\left(1-x_{0}\right)}$ is a quasi-eigenvalue of multiplicity 2 .

In the case $\mathcal{I}(\alpha)=0$, i.e., when $\alpha \in \mathbb{R} \backslash\left(-S^{-1 / 2}, S^{-1 / 2}\right)$, the infimum in (1.3) is not achieved. An optimizing sequence of strings those quasi-eigenvalues tend to $\alpha \in \mathbb{R} \backslash\left(-S^{-1 / 2}, S^{-1 / 2}\right)$ is provided by Proposition 2.3. Indeed, the strings $\mathrm{d} \Delta_{1-(2 \beta)^{-1}, 2 \beta\left(\alpha^{2}+\beta^{2}\right)^{-1}}$ belong to $A d$ for $\beta>0$ small enough and their sets of quasi-eigenvalues are $\{ \pm \alpha+\mathrm{i} \beta\}$.

On the other side, $K(A d)$ is a closed set in the relative topology of $\mathbb{C}_{+}$. So for $\alpha \in\left(-S^{-1 / 2}, S^{-1 / 2}\right)$ minimizers exist.

Theorem 2.4. Let $\alpha \in\left(-S^{-1 / 2}, S^{-1 / 2}\right)$ and let $\kappa^{[\alpha]}=\alpha+\mathrm{i} \mathcal{I}(\alpha)$ be the corresponding optimal quasieigenvalue. Then for the frequency $\alpha$ there exists a unique optimal string $\mathrm{d} M_{\alpha}$ (i.e., $\mathrm{d} M_{\alpha} \in$ Ad and $\left.\kappa^{[\alpha]} \in K\left(\mathrm{~d} M_{\alpha}\right)\right)$. The string $\mathrm{d} M_{\alpha}$ is of the form $\mathrm{d} \Delta_{x_{0}, A}$ and satisfies at least one of the equalities $\operatorname{Tm}_{M}=m, \operatorname{Stm}_{M}=S$.

More precisely, $\mathrm{d} M_{0}=\mathrm{d} \Delta_{1, m}$.

For $0<\alpha<S^{-1 / 2}, \mathrm{~d} M_{\alpha}=\mathrm{d} M_{-\alpha}=\mathrm{d} \Delta_{x_{0}, A}$ with $x_{0}$ and $A$ given by the following equalities:

when $S \leq m^{2} / 4, \quad x_{0}=1-\frac{1}{2\left(S^{-1}-\alpha^{2}\right)^{1 / 2}}, \quad A=2 S \sqrt{S^{-1}-\alpha^{2}}$;

when $S>m^{2} / 4, \quad\left(x_{0}, A\right)=\left\{\begin{array}{rr}\left(1-\frac{m}{2+2\left(1-\alpha^{2} m^{2}\right)^{1 / 2}}, m\right), & 0<\alpha<\sqrt{\frac{1}{S}-\frac{m^{2}}{4 S^{2}}} \\ \left(1-\frac{1}{2\left(S^{-1}-\alpha^{2}\right)^{1 / 2}}, 2 S \sqrt{S^{-1}-\alpha^{2}}\right), & \sqrt{\frac{1}{S}-\frac{m^{2}}{4 S^{2}}} \leq \alpha \leq S^{-1 / 2}\end{array}\right.$

Remark 2.2. The family $A d$ is a convex set in the space of signed Borel measures on $(-\infty, 1]$. Theorem 2.4 immediately implies that the strings $\mathrm{d} M_{\alpha}$ are extreme points of $A d$.

Denote by $K^{\text {mult }}(A d):=\bigcup\left\{K_{r}(\mathrm{~d} M): r \geq 2\right.$ and $\left.\mathrm{d} M \in A d\right\}$ the set of all possible non-simple quasi-eigenvalues for strings from $A d$. Using $\kappa^{[\alpha]}$ and $\mathrm{d} M_{\alpha}$ of Theorem 2.4, we state the following.

Theorem 2.5. Let $\alpha \in\left(-S^{-1 / 2}, S^{-1 / 2}\right)$. Then the optimal quasi-eigenvalue $\kappa^{[\alpha]}$ is a simple quasieigenvalue of the optimal string $\mathrm{d} M_{\alpha}$. Moreover, the distance from $\kappa^{[\alpha]}$ to $K^{\text {mult }}(A d)$ is positive.

Theorems 2.4 and 2.5 will be proved in Section 4 .

Remark 2.3. All the results of this subsection are valid if singular strings are included into the admissible family, i.e., if one replaces $\mathfrak{S}_{\text {fin }}$ with $\mathfrak{S}$ in $(1.2)$. The proofs do not require changes. 


\section{Tools.}

Denote $\ell:=1-a_{2}$, where $a_{2}$ is defined by (2.2). So $\ell$ is the length of a maximal interval $(x, 1]$ carrying Lebesgue's measure, $\ell=0$ exactly when the string is reduced. It is easy to see that $K(\mathrm{~d} M(x))=K(\mathrm{~d} M(x-\ell))$ (see e.g. [11, Sec. 3.1]); that is, if we delete the interval $\left(a_{2}, 1\right]$ from a non-reduced string and shift the new right endpoint to 1 , the obtained reduced string has the same quasi-eigenvalues.

All possible sets of quasi-eigenvalues for strings of the class $\mathfrak{S}$ were characterized in [9, 11].

Theorem 3.1 (Theorem 3.1 of [11]). Let $\left\{\kappa_{j}\right\}$ be an (empty, finite, or infinite) sequence of complex numbers, some of those may coincide. Then the set $\left\{\kappa_{j}\right\}$ is the set of quasi-eigenvalues (taking multiplicities into account) of a certain string $\mathrm{d} M \in \mathfrak{S}$ if and only if all the following conditions are fulfilled:

1) The set $\left\{\kappa_{j}\right\}$ is symmetric w.r.t. the imaginary axis $i \mathbb{R}$, moreover, the multiplicities of symmetric numbers are the same.

2) $\operatorname{Im} \kappa_{j}>0$ for all $j$, and $\sum_{j}\left|\operatorname{Im}\left(1 / \kappa_{j}\right)\right|<\infty$.

3) $\sum_{j}\left|\kappa_{j}\right|^{-2}<\infty$.

If these conditions are fulfilled, there exists a unique reduced string $\mathrm{d} M$ with the set of quasieigenvalues $\left\{\kappa_{j}\right\}$.

According to the last equality in [11, Sec.4.1],

$$
\sum_{j}\left|\operatorname{Im}\left(1 / \kappa_{j}\right)\right|=\operatorname{Tm}_{M}-\ell
$$

We use the following power series decomposition of $\varphi$ (see e.g. [11, Sec. 1.1])

$$
\begin{array}{r}
\varphi(x, \kappa)=1-\varphi_{1}(x) \kappa^{2}+\varphi_{2}(x) \kappa^{4}-\varphi_{3}(x) \kappa^{6}+\ldots, \\
\varphi_{0}(x) \equiv 1, \quad \varphi_{j}(x)=\int_{-\infty}^{x}(x-s) \varphi_{j-1}(s) \mathrm{d} M(s), \quad j \in \mathbb{N},
\end{array}
$$

to get a formula for the statical moment in terms of quasi-eigenvalues' positions, $\ell$, and $\operatorname{Tm}_{M}$.

\section{Proposition 3.2.}

$$
\begin{aligned}
\operatorname{Stm}_{M} & =\sum_{\operatorname{Re} k_{j}>0} \frac{1}{\left|\kappa_{j}\right|^{2}}+4 \sum_{\substack{\operatorname{Re} k_{j}, \operatorname{Re} k_{n}>0 \\
j \neq n}} \frac{\operatorname{Im} \kappa_{j} \operatorname{Im} \kappa_{n}}{\left|\kappa_{j}\right|^{2}\left|\kappa_{n}\right|^{2}}+2 \sum_{\substack{\operatorname{Re} k_{j}>0 \\
\operatorname{Re} k_{n}=0}} \frac{\operatorname{Im} \kappa_{j} \operatorname{Im} \kappa_{n}}{\left|\kappa_{j}\right|^{2}\left|\kappa_{n}\right|^{2}} \\
& +\sum_{\substack{\operatorname{Re} k_{j}=\operatorname{Re} k_{n}=0 \\
j \neq n}} \frac{\operatorname{Im} \kappa_{j} \operatorname{Im} \kappa_{n}}{\left|\kappa_{j}\right|^{2}\left|\kappa_{n}\right|^{2}}+\ell\left(\left.\operatorname{Tm}\right|_{M}-\frac{\ell}{2}\right) .
\end{aligned}
$$

Proof. In our notation, [11, formula (3.9)] takes the form

$$
\varphi(1, \kappa)-\mathrm{i} \partial_{x}^{+} \varphi(1, \kappa) / \kappa=e^{\mathrm{i} \kappa \ell} \prod_{\operatorname{Re} k_{j}>0}\left[\left(1-\frac{\kappa}{\kappa_{j}}\right)\left(1+\frac{\kappa}{\overline{\kappa_{j}}}\right)\right] \prod_{\operatorname{Re} k_{j}=0}\left(1-\frac{\kappa}{\kappa_{j}}\right) .
$$


(Note that our $\varphi(1, \kappa)$ is $\varphi\left(b, \kappa^{2}\right)$ of [11 and that the formula for $Q$ in [11] is given with a misprint, cf. [10] and also [8, pp. 303-6]).

Plugging (3.2) into the left side,

$$
\begin{gathered}
{\left[1-\kappa^{2} \varphi_{1}(1)+O\left(\kappa^{4}\right)\right]-\frac{\mathrm{i}}{\kappa}\left[-\kappa^{2} \partial_{x}^{+} \varphi_{1}(1)+O\left(\kappa^{4}\right)\right]=} \\
{\left[1+\mathrm{i} \kappa \ell-\kappa^{2} \ell^{2} / 2+O\left(\kappa^{3}\right)\right] \prod_{\operatorname{Re} k_{j}>0}\left[1-\kappa\left(\kappa_{j}^{-1}-\overline{\kappa_{j}^{-1}}\right)-\kappa^{2}\left|\kappa_{j}\right|^{-2}\right] \prod_{\operatorname{Re} k_{j}=0}\left(1-\kappa / \kappa_{j}\right),}
\end{gathered}
$$

and comparing the coefficient of $\kappa^{2}$, we get

$$
\begin{aligned}
-\varphi_{1}(1) & =-\sum_{\operatorname{Re} k_{j}>0} \frac{1}{\left|\kappa_{j}\right|^{2}}-4 \sum_{\substack{\operatorname{Re} k_{j}, \operatorname{Re} k_{n}>0 \\
j \neq n}} \frac{\operatorname{Im} \kappa_{j} \operatorname{Im} \kappa_{n}}{\left|\kappa_{j}\right|^{2}\left|\kappa_{n}\right|^{2}}-2 \sum_{\substack{\operatorname{Re} k_{j}>0 \\
\operatorname{Re} k_{n}=0}} \frac{\operatorname{Im} \kappa_{j} \operatorname{Im} \kappa_{n}}{\left|\kappa_{j}\right|^{2}\left|\kappa_{n}\right|^{2}} \\
& -\sum_{\substack{\operatorname{Re} k_{j}=\operatorname{Re} k_{n}=0 \\
j \neq n}} \frac{\operatorname{Im} \kappa_{j} \operatorname{Im} \kappa_{n}}{\left|\kappa_{j}\right|^{2}\left|\kappa_{n}\right|^{2}}-\frac{\ell^{2}}{2}-\ell \sum_{j} \frac{\operatorname{Im} \kappa_{j}}{\left|\kappa_{j}\right|^{2}}
\end{aligned}
$$

(the symmetry of $K(\mathrm{~d} M)$ w.r.t. i $\mathbb{R}$ was used to modify the last term). By (3.3), $\varphi_{1}(1)=\operatorname{Stm}_{M}$. By (3.1), the last term in (3.5) equals $\ell\left(\operatorname{Tm}_{M}-\ell\right)$. Taking into account these equalities, one can write (3.5) as (3.4).

Remark 3.1. Squaring (3.1) and taking again into account the symmetry of $K(\mathrm{~d} M)$ w.r.t. i $\mathbb{R}$, it is not difficult to notice that (3.4) can be written shorter:

$$
\operatorname{Stm}_{M}=\frac{1}{2} \operatorname{Tm}_{M}^{2}+\frac{1}{2} \sum_{j} \frac{\left(\operatorname{Re} \kappa_{j}\right)^{2}-\left(\operatorname{Im} \kappa_{j}\right)^{2}}{\left|\kappa_{j}\right|^{4}} .
$$

However (3.4) is more convenient for the needs of the next section.

\section{Proofs of Theorems 2.1, 2.4, and 2.5.}

Lemma 4.1. Let $\kappa=\alpha+\mathrm{i} \beta \in K(\mathrm{~d} M)$ and $\alpha \neq 0$. Then the following assertions hold.

(i) $\kappa \notin \mathbb{D}_{\mathrm{Tm}_{M}^{-1}}\left(\mathrm{iTm}_{M}^{-1}\right)$ and $\kappa \notin \mathbb{D}_{\mathrm{Stm}_{M}^{-1 / 2}}(0)$.

(ii) If $\kappa \in \mathbb{T}_{\mathrm{Tm}_{M}^{-1}}\left(\mathrm{iTm}_{M}^{-1}\right)$, then $K(\mathrm{~d} M)$ consists of two simple quasi-eigenvalues $\pm \alpha+\mathrm{i} \beta$ and $\mathrm{d} M=\mathrm{d} \Delta_{1-(2 \beta)^{-1}, \operatorname{Tm}_{M}}$.

(iii) If $\kappa \in \mathbb{T}_{\operatorname{Stm}_{M}^{-1 / 2}}(0)$, then $K(\mathrm{~d} M)$ consists of two simple quasi-eigenvalues $\pm \alpha+\mathrm{i} \beta$ and $\mathrm{d} M=\mathrm{d} \Delta_{x_{0}, A}$ with $\left(x_{0}, A\right)=\left(1-\frac{1}{2\left(\operatorname{Stm}_{M}^{-1}-\alpha^{2}\right)^{1 / 2}}, 2 \operatorname{Stm}_{M} \sqrt{\operatorname{Stm}_{M}^{-1}-\alpha^{2}}\right)$.

(iv) If $\kappa \in K_{r}(\mathrm{~d} M)$ (i.e., the multiplicity of $\kappa$ is $r$ ), then $\kappa \notin \mathbb{D}_{r \operatorname{Tm}_{M}^{-1}}\left(\mathrm{i} r \operatorname{Tm}_{M}^{-1}\right)$ and $\kappa \notin \mathbb{D} \sqrt{r \operatorname{Stm}_{M}^{-1}}(0)$.

Proof. If $\kappa \in K(\mathrm{~d} M)$, formula (3.1) and Theorem 3.1 (1) imply $\frac{2 \beta}{\alpha^{2}+\beta^{2}} \leq \operatorname{Tm}_{M}$. Theorem 3.1 (1) and (3.4) imply $\left(\alpha^{2}+\beta^{2}\right)^{-1} \leq \operatorname{Stm}_{M}$ (note that the definition of $\ell$ yields $\ell \leq \operatorname{Tm}_{M}$ ). These inequalities are equivalent to (i). Note that the equalities hold exactly when $K(\mathrm{~d} M)=\{ \pm \alpha+\mathrm{i} \beta\}$ and $\ell=0$. Combining this, Proposition 2.3, and the uniqueness statement of Theorem 3.1 one can easily get (ii) and (iii). In the case when $\kappa \in K_{r}(\mathrm{~d} M)$, (3.1) and (3.4) yield (iv). 
The case $\kappa \in \mathrm{i} \mathbb{R}$ is simpler, and the above arguments lead to the following lemma.

Lemma 4.2. Let $\mathrm{i} \beta \in K(\mathrm{~d} M)$. Then the following assertions hold.

(i) $\beta \geq \operatorname{Tm}_{M}^{-1}$.

(ii) If $\beta=\mathrm{Tm}_{M}^{-1}$, then $K(\mathrm{~d} M)$ consists of a single simple quasi-eigenvalue $\mathrm{i} \beta$ and $\mathrm{d} M=\mathrm{d} \Delta_{1, \mathrm{Tm}_{M}}$.

(iii) If $\mathrm{i} \beta \in K_{r}(\mathrm{~d} M)$, then $\beta \geq r \mathrm{Tm}_{M}^{-1}$.

Proof of Theorem 2.1. The inclusion $K(A d) \subset \mathrm{i}\left[m^{-1},+\infty\right) \cup\left(\mathbb{C}_{+} \backslash\left[\mathbb{D}_{m^{-1}}\left(\mathrm{i} m^{-1}\right) \cup \mathbb{D}_{S^{-1 / 2}}(0)\right]\right)$ follows immediately from Lemma 4.1 (i) and Lemma 4.2 (i). It is not difficult to obtain the inverse inclusion $K(A d) \supset \ldots$ from Proposition 2.3 considering all the strings of type $\mathrm{d} \Delta_{x_{0}, A}$ that belong to $A d$.

Proof of Theorem 2.4. Let $\alpha \in\left(-S^{-1 / 2}, S^{-1 / 2}\right)$. Then Corollary 2.2 yields $\mathcal{I}(\alpha)>0$. Theorem 2.1 implies that there exists at least one $\mathrm{d} M \in A d$ with a quasi-eigenvalue at $\kappa^{[\alpha]}$. In the case $\alpha \neq 0$, $\kappa^{[\alpha]}$ belongs to at least one of the circles $\mathbb{T}_{m^{-1}}\left(\mathrm{i} m^{-1}\right), \mathbb{T}_{S^{-1 / 2}}(0)$. By Lemma 4.1 (i), this is possible only if one of the bounds from the definition of $A d$ is reached. That is, only if one of the equalities $\mathrm{Tm}_{M}=m, \mathrm{Stm}_{M}=S$ hold. Now Lemma 4.1 (ii)-(iii) easily implies the statement of the theorem. The case $\alpha=0$ can be treated similarly with the use Lemma 4.2 .

Proof of Theorem 2.5. By Lemma 4.1 (iv), $K^{\text {mult }}(A d) \backslash \mathrm{i} \mathbb{R} \subset \mathbb{C}_{+} \backslash\left[\mathbb{D}_{2 m^{-1}}\left(\mathrm{i} 2 m^{-1}\right) \cup \mathbb{D}_{\sqrt{2 S^{-1}}}(0)\right]$. Lemma 4.2 (iii) yields $K^{\text {mult }}(A d) \cap \mathrm{i} \mathbb{R} \subset \mathrm{i}\left[2 m^{-1},+\infty\right)$. This easily implies the theorem.

\section{References}

[1] Y. Akahane, T. Asano, B. Song, S. Noda, High-Q photonic nanocavity in a two-dimensional photonic crystal, Nature 425 (2003) 944-947.

[2] D.Z. Arov, The realization of a canonical system with dissipative boundary conditions at one end of a segment in terms of the coefficient of dynamic flexibility, Sibirsk. Mat. Zh. 16 (1975), no.3, 440-463; English transl.: Siberian Math. J. 16 (1975), no.3, 335-352.

[3] S. Cox, E. Zuazua, The rate at which energy decays in a string damped at one end, Indiana Univ. Math. J. 44 (1995), no.2, 545-573.

[4] H. Dym, H.P. McKean, Gaussian Processes, Function Theory, and the Inverse Spectral Problem, Academic Press, New York, 1976.

[5] G.M. Gubreev, V.N. Pivovarchik, Spectral analysis of the Regge problem with parameters, Funktsional. Anal. i Prilozhen. 31 (1997), no.1, 70-74 (Russian); English transl.: Funct. Anal. Appl. 31 (1997), no. 1, 54-57.

[6] I.S. Kac and M.G. Krein, On the spectral functions of the string, Supplement II in Atkinson, F. Discrete and continuous boundary problems. Mir, Moscow 1968. Engl. transl.: Amer. Math. Soc. Transl., Ser. 2, 103 (1974) 19-102.

[7] M.G. Krein, On certain problems on the maximum and minimum of characteristic values and on the Lyapunov zones of stability, Prikl. Mat. Meh. 15 (1951) 323-348 (Russian); English transl.: Amer. Math. Soc. Transl. (2) 1 (1955) 163-187. 
[8] M.G. Krein, Selected works. I. Complex analysis, extrapolation, interpolation, Hermitianpositive functions and related topics, Akad. Nauk Ukrainy, Kiev, 1993 (Russian).

[9] M.G. Krein, A.A. Nudelman, On direct and inverse problems for the boundary dissipation frequencies of a nonuniform string, Dokl. Akad. Nauk SSSR 247 (1979), no. 5, 1046-1049 (Russian); English transl.: Soviet Math. Dokl. 20 (1979), no.4, 838-841.

[10] M.G. Krein, A.A. Nudelman, Representation of entire functions that are positive on the real axis, or on the half axis, or outside a finite interval, Mat. Issled. 61 (1981), 40-59 (Russian).

[11] M.G. Krein, A.A. Nudelman, Some spectral properties of a nonhomogeneous string with a dissipative boundary condition, J. Operator Theory 22 (1989) 369-395 (Russian).

[12] M.A. Naimark, Linear differential operators, second ed., Nauka, Moscow, 1969 (Russian); English transl.: Parts I, II. Frederick Ungar Publishing Co., New York, 1967-68.

[13] A.A. Shkalikov, Spectral analysis of the Redge problem. J. Math. Sci. 144 (2007), no. 4, 42924300 . 